\title{
Fundamental Cycles and Graph Embeddings 1
}

\author{
Ren Han2, Zhao Hongtao and Li Haoling \\ Dept. of Mathematics, East China Normal University \\ Shanghai 200062, P.R.China \\ E-mail: hren@math.ecnu.edu.cn
}

\begin{abstract}
In this paper, we investigate fundamental cycles in a graph $G$ and their relations with graph embeddings. We show that a graph $G$ may be embedded in an orientable surface with genus at least $g$ if and only if for any spanning tree $T$, there exists a sequence of fundamental cycles $C_{1}, C_{2}, \cdots, C_{2 g}$ with $C_{2 i-1} \cap C_{2 i} \neq \phi$ for $1 \leq i \leq g$. In particular, among $\beta(G)$ fundamental cycles of any spanning tree $T$ of a graph $G$, there are exactly $2 \gamma_{M}(G)$ cycles $C_{1}, C_{2}, \cdots, C_{2 \gamma_{M}(G)}$ such that $C_{2 i-1} \cap C_{2 i} \neq \phi$ for $1 \leq i \leq \gamma_{M}(G)$, where $\beta(G)$ and $\gamma_{M}(G)$ are, respectively, the Betti number and the maximum genus of $G$. This implies that it is possible to construct an orientable embedding with large genus of a graph $G$ from an arbitrary spanning tree $T$ ( which may have very large number of odd components in $G \backslash E(T)$ ). This is different from the earlier work of Xuong and Liu[9,6], where spanning trees with small odd components are needed. In fact, this makes a common generalization of Xuong[9],Liu[6] and $\mathrm{Fu}$ et al[2]. Further more, we show that (1).This result is useful in locating the maximum genus of a graph having a specific edge-cut. Some known results for embedded graphs are also concluded;(2). The maximum genus problem may be reduced to the maximum matching problem. Based on this result and the algorithm of MicaliVazirani[8], we present a new efficient algorithm to determine the maximum genus of a graph in $O\left((\beta(G))^{\frac{5}{2}}\right)$ steps. Our method is straight and quite deferent from the algorithm of Furst,Gross and McGeoch[3] which depends on a result of Giles[4]where matroid parity method is needed.
\end{abstract}

Keyword : Fundamental cycles, Maximum genus,upper-embedded . AMS 2000: Primary 05C10, secondary 05C70

\footnotetext{
${ }^{1}$ Supported by Natural Science Foundation of China ( Under the Granted Number 10271048, 10671073)

${ }^{2}$ This work is partially supported by Science and Technology Commission of Shanghai Municipality (07XD14011) and Shanghai Leading Academic Discipline Project, Project NumberB407
} 


\section{Definitions and Notations}

The graph considered here is finite and undirected and, furthermore, is connected unless it is stated otherwise. In general, multiple edges and loops are allowed. Terminology and notation without explicit explanation follows as from $[1,6,7]$.

By a surface, denoted by $S$, we mean a compact and connected 2-manifold without boundary. It is well known from elementary topology that surfaces can be divided into two classes: orientable and nonorientable ones. An orientable surface can be viewed as a sphere attached $h$ handles, while a nonorientable surface as a sphere attached $k$ crosscaps. The number $h$ or $k$ is called the genus of the surface. A cellular embedding of a graph $G$ into a surface $S$ is a continuous one-to-one mapping $\phi: G \rightarrow S$ such that each component of $G \backslash \phi(G)$ is homeomorphic to an open disc, called a face of $G$ ( with respect to this embedding $\phi$ ) and $\phi$ is called a cellular embedding( or embedding as some scholars called ). A cycle ( curve ) $C$ in an embedded graph in a surface $\sum$ is called surface separating if $\sum-C$ is disconnected. In particular, if $\sum-C$ has an open disc, denoted by $\operatorname{int}(C)$, then $C$ is called contractible (otherwise, $C$ is noncontractible), and $\operatorname{int}(C)+C=\operatorname{Int}(C)$ is the inner part of $C$. The other part of $\sum-C$ is called exterior of $C$ and is denoted by $\operatorname{Ext}(C)$.

Recall that the maximum genus $\gamma_{M}(G)$ of a graph $G$ is the largest integer $k$ such that $G$ has an embedding in an orientable surface with genus $k$. Since any graph $G$ embedded in a surface has at least one face, Euler's formula shows that $\gamma_{M}(G) \leq\left\lfloor\frac{\beta(G)}{2}\right\rfloor$, where $\beta(G)=|E(G)|-|V(G)|+1$ is known as Betti number of $G$ ( which is equal to the cyclic number of $G$ ). A graph is upper-embeddable if $\gamma_{M}(G)=\left\lfloor\frac{\beta(G)}{2}\right\rfloor$.

Let $G$ be a graph and $T$ be a spanning tree of $G$. It is clear that for any edge $e \in E(G) \backslash E(T), T+e$ contains a unique cycle of $G$, denoted by $C_{T}(e)$, which is called a fundamental cycle of $G$ ( with respect to the spanning tree $T$ of $G$ ). If a pair of edges $e_{1}$ and $e_{2}$ have a common end vertex in a graph $G$, then we say that the pair $\left\langle e_{1}, e_{2}\right\rangle$ is an adjacent-edge pair in G.Let $G_{1}$ and $G_{2}$ be a pair of disjoint subgraphs of $G$. Then $E\left[G_{1}, G_{2}\right]$ is the set of edges with their ends in $G_{1}$ and $G_{2}$,respectively.

Denote by $\xi(G, T)$ the number of components of $G \backslash E(T)$ with an odd number of edges. Then the Betti deficiency of $G$ denoted by $\xi(G)$ is defined as the value $\min _{T} \xi(G, T)$, where the minimum is taken over all spanning 
trees $T$ of $G$. A spanning tree $T$ of $G$ is said to be an optimal spanning tree if $\xi(G, T)=\xi(G)$.

In the following, the paper is organized as follows: in $\S 2$ we give a good characterization (i.e., Theorems 1 and 2) of maximum genus; $\S 3$ will concentrate on the applications of Theorems 1 and 2 and their refined form; $\S 4$ will show that finding the maximum genus of a graph $G$ is, in some extend, equivalent to the problem of finding a maximum matching in a specific graph $G_{M}$ called the fundamental intersecting graph of $G$ and presents an efficient algorithm in finding the maximum genus of a graph.

\section{A Good Characterization}

Lemma $1[6,9]$ Let $G$ be a graph, then

(1) $\gamma_{M}(G)=\frac{1}{2}(\beta(G)-\xi(G))$;

(2) $G$ is upper embeddable if and only if $\xi(G) \leq 1$.

Theorem 1.If a graph $G$ contains a spanning tree $T$ such that there exist $2 g$ fundamental cycles $C_{1}, C_{2}, \cdots, C_{2 g}$ with $C_{2 i-1} \cap C_{2 i-1} \neq \phi$, for $i=1,2 \cdots g$, then $G$ may be embedded in an orientable surface with genus at least $g$.

Proof Let $G$ and $T$ be as assumed and $e_{1}, e_{2}, \cdots, e_{2 g}$ be edges in $E(G) \backslash$ $E(T)$ such that $C_{i}$ is the unique cycle in $T+e_{i}(1 \leq i \leq 2 g)$. We may suppose further that $G=T+\left\{e_{1}, e_{2}, \cdots, e_{2 g}\right\}$ by Xuong's constructive proof of maximum genus formula[9]. Let $G_{0}=T$, and $G_{1}=G_{0}+\left\{e_{1}, e_{2}\right\}$. Then we have the following.

Claim 1. $\xi\left(G_{1}\right) \leq \xi\left(G_{0}\right)\left(\Leftrightarrow \gamma_{M}\left(G_{1}\right) \geq \gamma_{M}\left(G_{0}\right)+1\right)$.

To see this, we observe that $\beta\left(G_{0}\right)=\beta\left(G_{1}\right)-2$, and so, $\xi\left(G_{1}\right) \equiv$ $\xi\left(G_{0}\right)(\bmod 2)$.

If $\xi\left(G_{1}\right) \geq \xi\left(G_{0}\right)+2$, then we have one of the following situations:

(1). Both $e_{1}$ and $e_{2}$ have, respectively, their ends in distinct even components in $E\left(G_{0}\right) \backslash E(T)$ ( As shown in left hand side of Fig.1).

(2). Both $e_{1}$ and $e_{2}$ have, respectively, their ends in the same even components in $E\left(G_{0}\right) \backslash E(T)$ ( As shown in center of Fig.1).

(3). Exactly, one of $e_{1}$ and $e_{2}$, say $e_{1}$, joins two even components of $E\left(G_{0}\right) \backslash E(T)$, while $e_{2}$ has two ends in the same even components in $E\left(G_{0}\right) \backslash E(T)$ ( As shown in right hand side of Fig.1).

Without loss of generality, we may suppose that $e_{1} \cap e_{2}=\phi$, and consider 

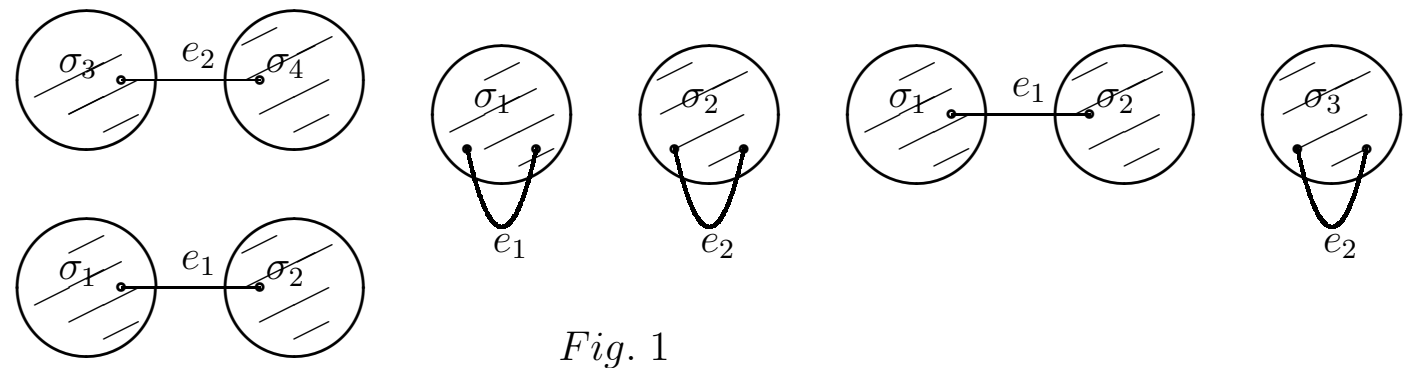

Fig. 1

case (1).

Let $e_{1} \in E\left[\sigma_{1}, \sigma_{2}\right], e_{2} \in E\left[\sigma_{3}, \sigma_{4}\right]$, and $C_{i}$ be the fundamental cycle in $T+e_{i}(1 \leq i \leq 2)$.

Subcase A. $C_{1} \cap C_{2}$ is a path.

Let $P=C_{1} \cap C_{2}$ be a path with an end vertex $x$ in $C_{1} \cap C_{2}$. Let $e_{1}^{\prime}$ and $e_{2}^{\prime}$ be two edges such that $e_{1}^{\prime}, e_{2}^{\prime} \in E(T)$, and $x \in e_{1}^{\prime} \cap e_{2}^{\prime}$. Now $e_{1}^{\prime}, e_{2}^{\prime} \notin E(P)$. (As shown in left hand side of Fig.2).Consider a new spanning tree $T^{\prime}=T+$ $\left\{e_{1}, e_{2}\right\}-\left\{e_{1}^{\prime}, e_{2}^{\prime}\right\}$.
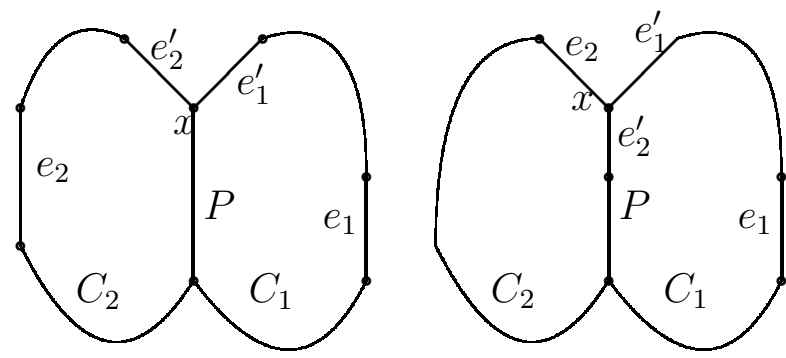

Fig.2

Subcase B. $x \in e_{1}$ or $x \in e_{2}$, say $x \in e_{2}$. (As shown in right hand side of Fig.2).

If $|E(P)| \geq 1$, then we take edges $e_{1}^{\prime} \in C_{1} \backslash E(T), x \in e_{1}^{\prime}, e_{2}^{\prime} \in E(P), x \in$ $e_{2}^{\prime}$. We construct a new spanning tree $T^{\prime}=T+\left\{e_{1}, e_{2}\right\}-\left\{\begin{array}{ll}e_{1}^{\prime}, & e_{2}^{\prime}\end{array}\right.$. If $|E(P)|=0$, then this may be a special case of $\mathrm{A}$.

Let $T^{\prime}$ be the spanning tree as defined in either subcase $\mathrm{A}$ or B. It is easy to see that $E\left(G_{1}\right) \backslash E\left(T^{\prime}\right)$ has at most $\xi\left(G_{0}\right)$ odd components . It is contradictory to our suppose. Therefore $\xi\left(G_{1}\right) \leq \xi\left(G_{0}\right)$.

Similarly, We may prove the claims in the cases of (2) and (3).

Repeat this procedure for $G_{2}=G_{1}+\left\{e_{3}, e_{4}\right\}, \cdots, G_{g}=G_{g-1}+\left\{e_{2 g-1}, e_{2 g}\right\}$ 
until we get $\xi\left(G_{g}\right) \leq \xi\left(G_{g-1}\right) \leq \cdots \leq \xi\left(G_{0}\right)$, so $\gamma_{M}\left(G_{g}\right) \geq \gamma_{M}\left(G_{0}\right)+g=g$.

Theorem 2. Let $G$ be a connected graph embedded in an orientable surface $S_{g}$ and $T$ be a spanning tree of $G$. Then there are at least $2 g$ noncontractible foundamental cycles $C_{1}, C_{2}, \cdots, C_{2 g}$, such that $C_{2 i-1} \cap C_{2 i} \neq \phi$ for $1 \leq i \leq$ $g$. In particular, if $G$ is a one-face-embedded graph in $S_{g}$, then for any spanning tree $T$ of $G$, there are $2 g$ edges in $G \backslash E(T)$ such that the corresponding $2 g$ fundamental cycles $C_{1}, C_{2}, \cdots, C_{2 g}$ satisfy $C_{2 i-1} \cap C_{2 i} \neq \phi$ for $1 \leq i \leq g$.

Proof. We contract $T$ into a single vertex $v_{T}$ and delete all the possible edges on distinct faces. Then we get a vertex-graph $G_{T}$ with exactly one vertex $v_{T}$ and one face in $S_{g}$. There are two crossed loops, say $e_{\alpha}, e_{\beta}$, such that the local rotation of semi-edges incident to $v_{T}$ is $e_{\alpha} \cdots e_{\beta} \cdots e_{\alpha} \cdots e_{\beta}$ ( as shown in Fig.3). Furthermore, $e_{\beta}$ is the only possible edge crossing $e_{\alpha}$ ( since otherwise $G_{T}$ would have at least two faces! ). Hence , all ( loop ) edges of $G_{T}$ may be listed as follows: $e_{1}, e_{2}, \cdots, e_{2 g-1}, e_{2 g}$ such that $e_{2 i-1}$ crossing $e_{2 i}$ for $i=1,2, \cdots, g$. It is easy to see that $e_{2 i-1}$ and $e_{2 i}$ determine two fundamental cycles $C_{2 i-1}$ and $C_{2 i}$ with a vertex in common. $\square$

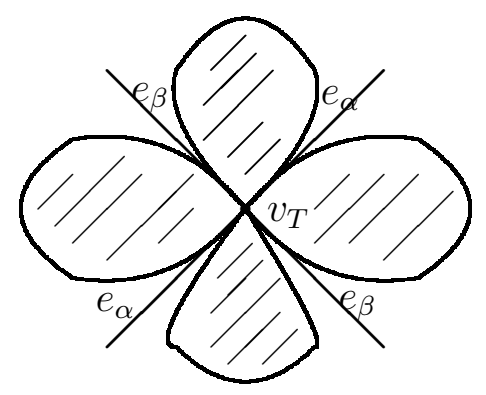

Fig.3

Remark: Theorems 1 and 2 give a good characterization of maximum genus of a graph(i.e., they implies the existence of a polynomially bounded algorithm to find the maximum genus of a graph).

Let $T$ be a spanning tree in $G$ with a group of fundamental cycles $C_{1}, C_{2}, \cdots, C_{2 g}$. If $C_{2 i-1} \cap C_{2 i} \neq \phi$ for $1 \leq i \leq g$, then we say $\left\langle C_{2 i-1}, C_{2 i}\right\rangle$ is an adjacent fundamental cycle pairs $(1 \leq i \leq g)$. If $g$ is chosen as the largest number satisfying above condition, then we call $g$ the maximum number of adjacent fundamental cycle pairs of $T$. Hence Theorem 2 implies the following:

Theorem 3 Any two spanning trees $T_{1}$ and $T_{2}$ in a graph $G$ have the same maximum number of adjacent fundamental cycle pairs. (In fact, this unique number is $\gamma_{M}(G)$, the maximum genus of $G$ ). 
This generalizes a result of $\mathrm{Fu}$ et al[2] where they introduced the concept intersecting graph which is determined by bases of cycle space of a graph to describe the maximum genus of a graph. In fact, our result stands for any spanning tree's fundamental cycles.

Corollary 1 If a connected graph $G$ has a spanning tree $T$ such that any two fundamental cycles have a vertex in common. Then $G$ is upper-embeddable.

Sometimes however, we need a refined form of Theorems 1 and 2 in practice. The following result gives us a recursive relation between the maximum genera of a graph and its subgraph(s).

Theorem 4 Let $G$ be a connected graph and $T$ be an arbitrary spanning tree in $G$. If $e_{1}, e_{2}$ are two edges not in $G$ and the two cycles $C_{T}\left(e_{1}\right)$ and $C_{T}\left(e_{2}\right)$ have a vertex in common. Then $\gamma_{M}(G)=\gamma_{M}\left(G+e_{1}+e_{2}\right)-1$. In particular, $G$ is upper-embeddable if and only if $G+e_{1}+e_{2}$ is upper-embeddable.

One may easily see that this generalizes a recursive relation for maximum genus of Xuong[9] and ( we will see in the next section )is much more practical in use.

\section{Applications}

Now in this section, we begin to apply Theorems $1-2$ to determine the maximum genus of some type of graphs.

Let us recall that the essence of Xuong's method[9] consists of two parts: one is to find an optimal tree $T$ in a graph $G$ having the smallest number of odd components; the other is to organize edges of $E(G) \backslash E(T)$ into adjacent pairs such as

$$
E(G) \backslash E(T)=\left\{e_{1}, e_{2}, \cdots, e_{2 s}\right\} \cup\left\{f_{1}, f_{2}, \cdots f_{m}\right\},
$$

where $e_{2 i-1} \cap e_{2 i} \neq \phi(1 \leq i \leq s)$ and $C_{T}\left(f_{i}\right) \cap C_{T}\left(f_{j}\right)=\phi$, for $1 \leq i<$ $j \leq m$ and $s=\gamma_{M}(G), m=\xi(G)$. Compared with the above procedure, Theorems 1 and 2 consider adjacent foundamental cycle pairs(rather than adjacent pairs of edges). We may construct large genus embedding from any spanning tree $T$, although it may have very large number of odd components in $G \backslash E(T)$. This greatly releases the conditions of Xuong. Of course, an optimal tree is also valid in our constructions. Hence, Theorems 1 and 2 
generalize Xuong's characterization of maximum genus. Based on this idea, we may construct a large orientable genus as follows: Take a specific spanning tree $T$ in graph $G$ and first organize some non-tree edges into adjacent pairs ( as Xuong did ) and then match other possible non-tree edges into pairs such that their fundamental cycles also become adjacent fundamental cycle pairs. It is easy for one to see that the second part of non-tree edges may be chosen as an edge-cut of $G$. Therefor, Theorems $1-2$ may be useful in determination of a maximum genus of a graph $G$ with a specific edge-cut. Now, the following result is easy to be verified.

Theorem 5 Let $A=\left\{e_{1}, e_{2}, \cdots, e_{k}\right\}$ be an edge-cut of $G$ such that $G-A$ has exactly two components $G_{1}$ and $G_{2}$. If both $G_{1}$ and $G_{2}$ are upperembeddable, then $\gamma_{M}(G) \geq\left\lfloor\frac{\beta(G)}{2}\right\rfloor-1$. Furthermore, if $G$ satisfies one of the following conditions, then $G$ is upper-embeddable:

(1). $\beta\left(G_{1}\right) \equiv \beta\left(G_{2}\right) \equiv 0(\bmod 2)$

(2). $|A| \equiv 1(\bmod 2)$ and $\beta\left(G_{1}\right)+\beta\left(G_{2}\right) \equiv 1(\bmod 2)$.

The next result is due to Huang. As a consequence of the above results, we will give another proof.

Theorem 6(Huang[5]) Let $G$ be a strongly embedded graph in an orientable surface $S_{g}$ (i.e., all facial walks are cycles ). If the dual graph $G^{*}$ of $G$ has a surface separating Hamiltonian cycle, then $G$ is upper-embeddable.

Proof We will show the existence of a spanning tree $T$ of $G$ satisfying the conditions in Theorems 1 and 2. Let $\mathcal{F}=\left\{f_{1}, f_{2}, \cdots f_{\varphi}\right\}$ be the face-set of $G$ and $C^{*}$ be a surface separating Hamiltonian cycle in $G^{*}$. Let $E\left(C^{*}\right)=\left\{e_{1}^{*}, e_{2}^{*}, \cdots e_{\varphi}^{*}\right\}$ and $e_{i}^{*}=\left(f_{i}, f_{i+1}\right)$ for $1 \leq i \leq \varphi$. Let $e_{i}$ be the edge in $\partial f_{i} \cap \partial f_{i+1}$ corresponding to $e_{i}^{*}$ for $1 \leq i \leq \varphi$ (where $\partial f_{i}$ denotes the boundary of $f_{i}$ ).

Claim 2. $G-\left\{e_{1}, e_{2}, \cdots e_{\varphi-1}\right\}$ is a one-face embedded subgraph of $G$ in $S_{g}$, Furthermore, $G-\left\{e_{1}, e_{2}, \cdots e_{\varphi}\right\}$ has exactly two components $G_{1}$ and $G_{2}$.

Now $E\left[G_{1}, G_{2}\right]=\left\{e_{1}, e_{2}, \cdots e_{\varphi}\right\}$. Let $G_{1} \subset \operatorname{Int}\left(C^{*}\right)$ and $G_{2} \subset \operatorname{Ext}\left(C^{*}\right)$ and $\partial f_{i}$ denotes the boundary cycle of $f_{i}$ for $1 \leq i \leq \varphi$. Then we may construct a graph as follows. $H_{0}=\left(\partial f_{1} \cup \partial f_{2} \cup \cdots \cup \partial f_{\varphi-1}\right) \backslash\left\{e_{1}, e_{2}, \cdots e_{\varphi-1}\right\}$. It is easy to see that $H_{0}$ is a connected spanning subgraph of $G-\left\{e_{1}, e_{2}, \cdots e_{\varphi-1}\right\}$. (Hence, a spanning subgraph of $G)$. Let $e_{\varphi}=(\alpha, \beta)$ with $\alpha \in V\left(G_{1}\right), \beta \in V\left(G_{2}\right)$. Then $H_{0}-e_{\varphi}$ has exactly two components $H^{\prime}, H_{1}$ with $H^{\prime}=G_{1}$.

Claim 3. If $H_{1}$ has a cycle $C$, then $C$ must be a noncontractible cycle. 
This follows from the fact that $S_{g}-H_{0}$ has only one component. If $H_{1}$ has a cycle $C_{1}$, then delete an edge $e_{1}^{\prime} \in C_{1}$ and get a subgraph $H_{2}$ of $H_{1}$ with $V\left(H_{2}\right)=V\left(H_{1}\right)$. Repeat this procedure until we arrive at a connected subgraph $H_{k}$ of $H_{1}$ with $V\left(H_{k}\right)=V\left(H_{1}\right)$ and $H_{k}$ has no cycle.

Claim 4: $T=H^{\prime} \cup H_{k} \cup\left\{e_{\varphi}\right\}$ is a spanning tree of $G$, such that each fundamental cycle $C_{T}\left(e_{i}\right)$ in $T+e_{i}$ has an edge $e_{\varphi}$ in common for $i=1,2, \cdots, \varphi-1$.

To see this, we consider an edge $e_{i}=\left(x_{i}, y_{i}\right) \in\left[H^{\prime}, H_{k}\right] \subseteq\left[G_{1}, G_{2}\right]$, such that $x_{i} \in H^{\prime}, y_{i} \in H_{k}$. Since $H^{\prime}\left(H_{k}\right)$ is connected, there is a path $P_{i}\left(Q_{i}\right)$ in $H^{\prime}\left(H_{k}\right)$ joining $\alpha(\beta)$ and $x_{i}\left(y_{i}\right)$. Hence, $C_{T}\left(e_{i}\right)=\left\{e_{\varphi}\right\} \cup P_{i} \cup Q_{i} \cup\left\{e_{i}\right\}$ is a cycle containing $e_{\varphi}$ for $1 \leq i \leq \varphi$.

Now we find a spanning tree $T$ of $G$ such that:

(1). All the fundamental cycles $C_{T}\left(e_{1}\right), C_{T}\left(e_{2}\right), \cdots, C_{T}\left(e_{\varphi-1}\right)$ has an edge in common;

(2). By Theorems 1 and 2, and the fact that $T$ is also a spanning tree in $G-$ $\left\{e_{1}, e_{2}, \cdots e_{\varphi-1}\right\}$, there are another group of fundamental ( noncontractible ) cycles $C_{1}, C_{2}, \cdots, C_{2 g}$ such that $C_{2 i-1} \cap C_{2 i} \neq \varnothing$ for $1 \leq i \leq g$. By theorem $1, G$ is upper-embeddable.

One may readily see that a surface separating cycle may not be Hamiltonian and the hosting surface on which a graph is embedded may not be orientable. Thus, Theorem 6 can be extended to a much more generalized form.

Theorem 7 Let $G$ be an embedded graph in a surface $\sum$ such that the dual graph $G^{*}$ of $G$ contains a surface separating cycle $C^{*}$ such that both of the left subgraph $G_{L}\left(C^{*}\right)$ and right subgraph $G_{R}\left(C^{*}\right)$ of $G$ are upper embeddable . Then $\gamma_{M}(G) \geq\left\lfloor\frac{\beta(G)}{2}\right\rfloor-1$. In particular, if $\beta\left(G_{L}\left(C^{*}\right)\right) \equiv \beta\left(G_{R}\left(C^{*}\right)\right) \equiv$ $0(\bmod 2)$, then $G$ is upper-embeddable.

Remark: The term "left( right ) subgraph" follows from [7]

Corollary 2 If $G$ is an embedded graph on the Klein bottle such that the dual graph $G^{*}$ has a surface separating Hamiltonian cycle. Hence $\gamma_{M}(G) \geq$ $\left\lfloor\frac{\beta(G)}{2}\right\rfloor-1$.

In practical use, our attentions need not to be restricted to graphs with an edge-cut. Theorems 1-4 provide us a tool to evaluating large genus embeddings in more extended range of graphs. The following results show us 
how to do so ( we omit the proof of them ).

Theorem 8. The following graphs are upper-embeddable:

(1). The cartisian product $G \times P_{n}$ of a simple connected graph $G$ and a path $P_{n}$ with $n(\geq 1)$ egdes;

(2). The composition of two disjoint Halin graphs $H_{1}$ and $H_{2}$ with some edges $e_{1}, e_{2}, \cdots, e_{k}(k \geq 2)$ connecting them;

(3). The $n$-cube $Q_{n}$ which is composed of two $(n-1)$-cube $Q_{n-1}$ together with some edges joining the two copies of vertice in $Q_{n-1}$.

(4). The generalized Petersen graphs $P(n, k)$ which is determined by $n$-cycle $\left(u_{1}, u_{2}, \cdots, u_{n}\right)$ and vertices $v_{1}, v_{2}, \cdots, v_{n}$ such that $(i)$ each $\left(u_{i}, v_{i}\right) \in E, 1 \leq$ $i \leq n ;(i i)\left(u_{i}, v_{i+k}\right) \in E, 1 \leq i \leq n$.

Note: A graph $G=(V, E)$ is a Halin graph if $G$ is obtained by joining the leaves(1-valent vertices) of a plane tree $T$ with a cycle in this orientation and the definition of cartisian product of two graphs may be fund in and textbook of graph theory.

\section{A polynomially bounded algorithm}

In this section we shall present a polynomially bounded algorithm to find the maximum genus of a given graph. A basic fact is that Theorems 1 and 2 present a good characterization of maximum genus problem, i.e., we have the following

Theorem 9 To determine the maximum genus of a graph $G$ is equivalent to determine a maximum matching of the graph $G_{M}=\left(V_{M}, E_{M}\right)$, called fundamental intersecting graph of $G$, where $V_{M}$ is the set of fundamental cycles of a spanning tree $T$ of $G$ and any two cycles in $V_{M}$ are adjacent if and only if they have at least a vertex in common.

We observe that the fastest algorithm to find a maximum matching in a graph $G$ is due to Micali-Vazirani[8] which will end in $O(m \sqrt{n})$ steps, where $m$ and $n$ are, respectively, the number of edges and vertices of $G$. Based on this fact and Theorems 1 and 2 we may construct a new algorithm to determine the maximum genus of a graph $G$.

Fundamental cycle algorithm

Step 1.Input the date of the graph $G$ and then searching for a span- 
ning tree $T$ and the set $V_{M}$ of fundamental cycles in $G$;

Step 2. For cycles in $V_{M}$ we build the graph $G_{M}$;

Step 3 Perform Micali-Vazirani algorithm to find a maximum matching in $G_{M}$ and then terminate.

Remark: Since the number of fundamental cycles in a graph $G$ of order $n$ is $(\beta(G))$, this algorithm will end in at most $O\left((\beta(G))^{\frac{5}{2}}\right)$ steps. Although Furst, Gross and McGeoch had already construct the first polynomially bounded algorithm[3], this result is a new approach to do so.

\section{References}

[1] J.A. Bondy, U.S.R Murty, Graph Theory with Application, MacMillan, London, 1976.

[2] Hung-Lin Fu, Hsinchu, M. Skoviera, Bratislava, and M.Tsai, The maximum genus, matchings and the cycle space of a graph, Csechoslovak Math. J., 48(123)(1998),329-339

[3] M.L.Furst, J.L.Gross,L.A.McGeoch, Finding a maximum-genus graph imbedding, J.Assoc.Comput.Mach.35(1988), 523 - 534.

[4] R.Giles, Optimum matching forest I:Special weights, Math.Programming 22(1982), 1 - 11.

[5] Y. Huang, Maximum genus of a graph in term of its embedding properties, Discreate Math. 262 (2003) 171 - 180.

[6] Y.P.Liu The maximum orientable genus of a graph(Chinese with English abstract). Scientia Sinica, Special Issue on Math.II,, 41-55 (1979)

[7] B.Mohar, C.Thomassen. Graphs on Surface, Johns Hopkins University Press ,2001.

[8] S.Micali, V.V.Vazirani, An $O(\sqrt{|V|}|E|)$ algorithm for finding maximum matching in general graphs. In Proc.21th IEEE Symp.Found.Comp.Sci. $\operatorname{ACM}(1980), 17-27$

[9] N.H. Xoung, How to determine the maximum genus of a graph, J.Combin. Theory Ser.B 26 (1979) 217 - 225. 